ORIGINAL ARTICLE

\title{
Nurse management of intractable functional constipation: a randomised controlled trial
}

\author{
C A Burnett, E Juszczak, P B Sullivan
}

Arch Dis Child 2004;89:717-722. doi: 10.1136/adc.2002.025825

See end of article for authors' affiliations .....................

Correspondence to: Dr P B Sullivan, University of Oxford, Department of Paediatrics, John Radcliffe Hospital, Oxford OX3 9DU, UK; peter.sullivan@ paediatrics.ox.ac.uk

Accepted 29 September 2003

\begin{abstract}
Aims: To evaluate the effectiveness of a nurse led clinic (NLC) compared with a consultant led paediatric gastroenterology clinic (PGC) in the management of chronic constipation.

Methods: Children (age 1-15 years) with functional constipation were randomised following a detailed medical assessment to follow up in either the NLC or PGC. An escalating algorithm of treatment was used as the basis of management in both the NLC and PGC. Main outcome measures were: time to cure at last visit or later confirmed by telephone; time to cure at last visit; and time to prematurely leaving the study. Results: A total of 102 children were recruited, of whom 52 were randomly assigned to NLC and 50 to PGC. Outcome assessment showed that 34 children in the NLC and 25 children in the PGC were confirmed cured at their last visit or later confirmed by telephone. The median time to cure was 18.0 months in the NLC and 23.2 months in the PGC. The probability of being cured was estimated as $33 \%$ higher in the NLC compared to PGC (hazard ratio 1.33). Attending the NLC hastened time to cure by an estimated $18.4 \%$.

Conclusion: Children who attend an NLC are equally as, if not more likely to be cured of intractable constipation, than those attending a PGC and on average their cure will occur sooner. Results suggest that an NLC can significantly improve follow up for children with intractable constipation and highlight the important role for clinic nurse specialists in management of children with gastrointestinal disease.
\end{abstract}

$C^{1}$ hildhood constipation is a common problem accounting for approximately $4 \%$ of referrals to general

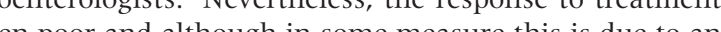
interaction of psychosocial influences and the pathophysiological effects of prolonged faecal retention, ${ }^{2-4}$ it is also possible that inadequate management and follow up contribute to an unsatisfactory outcome. In the follow up study of Keuzenkamp-Jansen and colleagues ${ }^{5}$ for example, $69 \%$ of children were still receiving laxatives at discharge and after one year's treatment, and 39\% of patients had recurrent symptoms four years after discharge. In another long term study symptoms of chronic constipation persisted in one third of patients nearly seven years after initial treatment. ${ }^{6}$

The objectives of this study were to: (1) evaluate the effectiveness of a nurse led clinic for management of chronic constipation in children; and (2) show non-inferiority of a nurse led clinic when compared to a paediatric gastroenterology clinic.

Nurse led services provide an ideal opportunity for nurses to advance their practice and take on greater responsibilities while educating others. ${ }^{7-9}$ The introduction of A\&E nurse practitioners, for instance, not only reduced the number of professionals coming into contact with the children but included other benefits. These include: better health education, prompt treatment for minor injuries, choice for children and families, and a reduced waiting time due to more efficient utilisation of staff. ${ }^{10} \mathrm{~A}$ recent report from a joint working party convened by the Royal College of Paediatrics and Child Health and the Joint Advisory Committee on Children's Nursing concluded that there is an urgent need for detailed, quantitative research into the health outcomes and continuity of care provided by nurses. ${ }^{11}$ This study, which aimed to show the effectiveness of a nurse led clinic for children with constipation, is a further contribution towards this end.

\section{METHODS}

Study design

This was a parallel group, randomised controlled noninferiority trial for which all children between the ages of 1 and 15 years presenting to the paediatric gastroenterology service at the John Radcliffe Hospital, Oxford, UK with constipation were potentially eligible. Our definition of constipation was: (1) decreased frequency of bowel movements (that is, decreased from the individual's previous pattern); and/or (2) harder stool consistency; and/or (3) subjective difficulty, including pain and distress associated with defecation. Children in whom organic or neurological disease had been identified were excluded. After initial assessment in the paediatric gastroenterology clinic, and after obtaining informed consent from parents, children were randomly assigned to follow up in either the nurse led clinic (NLC) or in the paediatric gastroenterology clinic (PGC). Allocation concealment was facilitated by using sequentially numbered sealed envelopes produced by an external source for consecutive and eligible study patients. The randomisation was performed using block randomisation with fixed blocks of size four. The Central Oxford Research Ethics Committee granted ethical approval for the study protocol.

\section{The nurse led clinic}

The nurse led clinic was designed to be a follow up clinic for children who had undergone a full and detailed medical assessment in the paediatric gastroenterology clinic leading

Abbreviations: AFT, accelerated failure time model; NLC, nurse led clinic; PGC, paediatric gastroenterology clinic 
to a diagnosis of idiopathic functional constipation according to the definition cited above. The report Developing roles of nurses in clinical child health ${ }^{11}$ also recommended that clinical practice should be based on clinical guidelines or protocols which have been agreed among the relevant professionals. Accordingly, an evidence based treatment algorithm for the management of childhood constipation, which is similar to a number of published guidelines, ${ }^{12-14}$ was constructed. Permission was given by the hospital's Drug and Therapeutics Committee, on the basis of the detailed algorithm, which also included recommended dosing regimes, for nurse prescribing of common laxative medications.

\section{Intervention}

This standardised treatment algorithm provided the basis for management decisions in all consultations in both the nurse led clinic and the paediatric gastroenterology clinic. The initial phases in this algorithm involved child and parent education about diet (fibre and fluid), exercise, toilet training, and the actions of the laxatives prescribed. Laxative therapy comprised a combination of stool softeners (for example, lactulose, docusate sodium) and stimulants. Stimulants of different potencies (senna, bisacodyl, sodium picosulphate) were prescribed according to the clinical response as indicated by the bowel diaries (see below). If there was an inadequate clinical response to this initial phase, the patient moved on to an advanced treatment regime which might include, enemas, intestinal lavage, manual removal of faeces under general anaesthesia, or psychological referral as was appropriate in each case.

Where it was clinically appropriate, an abdominal radiograph was obtained at the time of initial assessment both as a diagnostic tool and as a semi-quantitative marker of the severity of constipation. The validated scoring system ${ }^{15}$ used scores ranging from 0 (no stool) to 5 (gross faecal loading with bowel dilatation) in three areas of the colon, giving a total severity score ranging from 0 to 15 . Using this system a radiographic score of $\geqslant 9$ has been shown to have a high specificity and sensitivity in the diagnosis of childhood constipation.

Bowel diaries, which report the frequency, size, and consistency of stools, presence or absence of soiling, and a record of daily laxative medication, were used in both clinics to monitor progress and response to treatment. Dedicated case report forms were used for each study participant and, together with detailed clinical history (including a detailed dietetic history) and clinical findings on initial assessment, documented details of bowel habit and drug therapy at all subsequent outpatient visits. Any other contact with the families, for example on the telephone or a home visit, was documented using inter-visit contact forms.

A child was defined as having been "cured" of their constipation when, for a period of at least one month, they had been opening their bowels, producing a normal formed stool without difficulty at least three times per week and without any laxative therapy.

The primary outcome measures included:

(la) Time to cure at last visit or later confirmed by telephone. This outcome relates to all those children confirmed cured either at their last visit, or subsequently, confirmed over the telephone. Clinically this is the most relevant and important outcome since it mirrors clinical practice. Children who were close to achieving the definition of "cured" at their last visit but who were still being weaned off medication, were not required to attend for a further follow up appointment but received their follow up via the telephone. (lb) Time to cure at last visit. This outcome relates to only those children confirmed cured at their last visit (a subset of the previous outcome).

(2) Premature study termination. This outcome comprises those patients who are either lost to follow up or withdrawn for whatever reason. This is a negative outcome since it is a proxy measure of the ability of the clinical setting to facilitate continued follow up.

Secondary outcomes included:

(3) The number of clinic visits.

(4) The number requiring additional medication/in-patient procedures during the scheduled treatment period.

\section{Statistical analysis}

The primary outcome of cure at last visit or later confirmed by telephone was used to assess sample size. For non-inferiority to be concluded between NLC and PGC, 200 patients ( 100 per arm) would be required for a power of $80 \%$ and a one-sided significance level of 0.05 , assuming the success rate of the PGC to be $50 \%$. The range of clinical equivalence was defined to be within $15 \%$, therefore non-inferiority was defined as the ruling out of a hazard ratio less than 0.85 on the basis of the lower limit of the one sided $95 \%$ confidence interval. Conversely, for an outcome where a reduction of events is preferable, non-inferiority is defined as the ruling out of a hazard ratio greater than 1.176 on the basis of the upper limit of the one sided $95 \%$ confidence interval.

For all outcomes, the data were analysed comparing all those children allocated to NLC versus all those allocated to PGC-that is, on an intention-to-treat basis. Statistical analysis was conducted using SPSS for Windows (SPSS Inc., Chicago, IL, 2001) and STATA (StataCorp, College Station, TX, 2001).

For the primary time-to-event outcomes, the clinics were compared using survival analysis. This was performed in three ways: (1) graphically using reverse Kaplan-Meier plots (showing the cumulative percentage cured through time); (2) using Cox's proportional hazards model, allowing estimated treatment effects to be expressed as hazard ratios with associated one sided 95\% confidence intervals; and (3) using an accelerated failure time model (AFT), allowing estimated treatment effects to be expressed as time ratios (with associated one sided $95 \%$ confidence intervals). For the latter, the survival times were assumed to follow a logNormal distribution. This was verified by comparing observed and predicted survival curves, inspection of Q-Q plots, and Akaike's information criterion to compare the fit to that of other survival models.

A subsidiary analysis involved the tabulation and comparison of dichotomous outcomes relating to prespecified additional medication/procedures during the scheduled treatment period by treatment group. The statistical comparison used the Pearson $\chi^{2}$ test.

\section{RESULTS}

\section{Patients}

A total of 102 children were recruited to the study between February 1998 and December 2000, of whom 52 were randomly assigned to NLC and 50 to PGC. Baseline demographic and clinical presentation characteristics were well balanced across the clinics (table 1). Median follow up of 16.6 months was identical in the two groups. The median number of visits in each clinic was six. The median number of inter-visit contacts made to the NLC was 6.0 (range 2-16) and the median value for the PGC was 0.0 (range 0.0-29). 


\begin{tabular}{|c|c|c|}
\hline & $\begin{array}{l}\text { NLC } \\
(n=52)\end{array}$ & $\begin{array}{l}\text { PGC } \\
(n=50)\end{array}$ \\
\hline \multicolumn{3}{|l|}{ Demographics } \\
\hline Age, median ( $\min$ to $\mathrm{max}$ ), $\mathrm{mth}$ & $55.5(13-164)$ & $58(24-175)$ \\
\hline Male & $30(57.7 \%)$ & $25(50.0 \%)$ \\
\hline Female & $22(42.3 \%)$ & $25(50.0 \%)$ \\
\hline Family history & $19(36.5 \%)$ & $14(28.0 \%)$ \\
\hline Toilet trained & $36(69.2 \%)$ & $34(68.0 \%)$ \\
\hline $\begin{array}{l}\text { Duration of constipation, } \\
\text { median (min to max), mth } \\
\text { Clinical presentation }\end{array}$ & 25 (3-144) & $24(1-144)$ \\
\hline Clinical presentation & $42(80.8 \%)$ & $39(78.0 \%)$ \\
\hline $\begin{array}{l}\text { Bowels open per week, median } \\
\text { (min to max) }\end{array}$ & $2(1-14)$ & $2(0-14)$ \\
\hline Hard stools & $38(73.1 \%)$ & $39(72.0 \%)$ \\
\hline Difficulty with defecation & $48(92.3 \%)$ & $43(86.0 \%)$ \\
\hline \multicolumn{3}{|l|}{ Soiling } \\
\hline None & $22(42.3 \%)$ & $26(52.0 \%)$ \\
\hline Mild (×2/week) & $6(11.5 \%)$ & $6(12.0 \%)$ \\
\hline Moderate ( $\times 3 /$ week) & $5(9.6 \%)$ & $4(8.0 \%)$ \\
\hline Severe ( $\times 4 /$ week) & $19(36.5 \%)$ & $14(28.0 \%)$ \\
\hline Encopresis & $1(1.9 \%)$ & $4(8.0 \%)$ \\
\hline Anal fissure & $3(5.8 \%)$ & $6(12.0 \%)$ \\
\hline Enuresis & $7(13.5 \%)$ & $5(10.0 \%)$ \\
\hline Recurrent urinary tract infection & $1(1.9 \%)$ & $1(2.0 \%)$ \\
\hline \multicolumn{3}{|l|}{ Discomfort on defecation } \\
\hline None & $9(17.3 \%)$ & $14(28.0 \%)$ \\
\hline Mild & $13(25.0 \%)$ & $8(16.0 \%)$ \\
\hline Moderate & $15(28.8 \%)$ & $18(36.0 \%)$ \\
\hline Severe & $15(28.8 \%)$ & $10(20.0 \%)$ \\
\hline Withholding behaviour & $32(61.5 \%)$ & $29(58.0 \%)$ \\
\hline Fibre intake recorded as "poor" & $36(69.2 \%)$ & $41(82.0 \%)$ \\
\hline Abdominal $x$ ray score $(>9 / 15)$ & 33 out of 35 & 30 out of 31 \\
\hline
\end{tabular}

The median age at study entry was 4.6 years and 4.8 years in the NLC and PGC respectively. The youngest child in the study was 13 months, and the oldest was 14 years 7 months old; there were 55 males and 47 females. Overall, a family history of constipation was common and identified in a third (33/102) of all of children. Dietary fibre intake was reported by the parent/carer to be "poor" or "very poor" in three quarters (77/102) of the children. On average, the children had been constipated for a period of two years before they were referred. Three quarters (77/102) reported hard stools and 91/102 (89\%) reported difficulty with defecation; the median number of bowel openings per week was 2 . Clinically significant constipation was confirmed radiologically in 63/66 cases (95\%) for which radiological studies were requested. Urinary tract problems were found in 14/ 102 cases $(13.7 \%)$, a figure identical to that reported by Abrahamian and Lloyd-Still. ${ }^{2}$

A total of 10 children (five boys and five girls) completed the study as per the protocol but were not cured-that is, they are treatment failures, five in the NLC and five in the PGC. Eight of 10 children were formally referred for psychological/psychiatric management and 9/10 had documented serious behavioural problems. Three of 10 children were also referred for surgical assessment and management.

In this study, soiling (defined as the involuntary passage of faeces due to overflow incontinence secondary to rectal faecal impaction) was differentiated from encopresis (defined as the voluntary/involuntary passage of a normal bowel movement in an inappropriate place without an organic cause). Presentation with encopresis was relatively uncommon at 5/ $102(5 \%)$ whereas faecal soiling was very common, being reported in more than half of the cases (54/102), and was usually of a severe degree (defined as four or more times per week). Voluntary retention or withholding of stool was identified from the history in 61/102 children (60\%).
Table 2 Previous therapy

\begin{tabular}{lll}
\hline Type of therapy & $\begin{array}{l}\text { NLC } \\
(\mathbf{n = 5 2 )}\end{array}$ & $\begin{array}{l}\text { PGC } \\
\text { (n=50) }\end{array}$ \\
\hline Therapy & $50(96.2 \%)$ & $47(94.0 \%)$ \\
Lactulose & $50(96.2 \%)$ & $42(84.0 \%)$ \\
Senna & $39(75.0 \%)$ & $34(68.0 \%)$ \\
Docusate & $14(26.9 \%)$ & $10(20.0 \%)$ \\
Picolax & $14(26.9 \%)$ & $10(20.0 \%)$ \\
Suppositories & $6(11.5 \%)$ & $8(16.0 \%)$ \\
Bisacodyl & $3(5.8 \%)$ & $3(6.0 \%)$ \\
Enema(s) & $2(3.8 \%)$ & $6(12.0 \%)$ \\
Movicol & $2(3.8 \%)$ & $0(0.0 \%)$ \\
Klean-prep & $1(1.9 \%)$ & $2(4.0 \%)$ \\
Liquid paraffin & $1(1.9 \%)$ & $1(2.0 \%)$ \\
\hline
\end{tabular}

Given the relatively long period of constipation before referral (median 2 years), it is not surprising that nearly all children, 97/102 (95\%), had already received some form of laxative therapy. Table 2 shows the details and the similar distribution of prior laxative usage between the children in the two clinics.

Using bowel charts to monitor progress, less than half of the children responded solely to the initial treatment regime and therefore progressed to the advanced treatment regime. Details are shown in table 3; there was no difference in the proportion of children progressing to advanced treatment between the NLC and the PGC.

Children were followed up for a minimum of two years in order to complete the study protocol. A total of 15/102 children are still undergoing follow up, as they are not cured. In this group, 7/15 children are followed up in the PGC and 8/ 15 in the NLC. Seven of 15 children had documented psychosocial problems associated with poor compliance in attending clinic appointments.

\section{Efficacy outcomes}

The primary outcome measures used to detect differences in efficacy between the NLC and PGC included: (la) time to cure at last visit or later confirmed by telephone; ( $\mathrm{lb}$ ) time to cure at last visit; and (2) time to prematurely leaving the study.

Fifty nine children were confirmed to be cured at last visit or later confirmed by telephone; of these, 49 were confirmed to be cured at the last visit (table 4). Of those children cured at their last clinic visit or confirmed by subsequent telephone follow up, 34 of 52 (65.4\%) were cured in the NLC and 25 of $50(50.0 \%)$ in the PGC. The median time to cure was 18.0 months (95\% CI 8.5 to 27.5 ) in the NLC and 23.2 months (95\% CI 17.3 to 29.2 ) in the PGC. The probability of cure was estimated as $33 \%$ higher in the NLC compared to the PGC (hazard ratio 1.33 , one sided $95 \%$ CI 0.86 to $\infty, p=0.3$ ). The lower limit of the $95 \%$ confidence interval is greater than the minimum value defined as clinically equivalent-that is,

Table 3 Occurrence of prespecified advanced treatment regime

\begin{tabular}{llll}
\hline $\begin{array}{l}\text { Type of advanced } \\
\text { therapy }\end{array}$ & $\begin{array}{l}\text { NLC } \\
(\mathbf{n = 5 2 )}\end{array}$ & $\begin{array}{l}\text { PGC } \\
(\mathbf{n}=50)\end{array}$ & $\begin{array}{l}\chi^{2} \text { test } \\
\text { p value }\end{array}$ \\
\hline $\begin{array}{l}\text { Advanced therapy } \\
\text { Poly-ethylene glycol }\end{array}$ & $24(46.2 \%)$ & $29(58.0 \%)$ & 0.23 \\
lavage & $20(38.5 \%)$ & $19(38.0 \%)$ & 0.96 \\
Enema(s) & $22(42.3 \%)$ & $25(50.0 \%)$ & 0.44 \\
$\begin{array}{l}\text { Psychology referral } \\
\text { EUA and rectal biopsy }\end{array}$ & $12(23.1 \%)$ & $12(24.0 \%)$ & 0.91 \\
\hline
\end{tabular}




\begin{tabular}{|c|c|c|c|c|}
\hline Outcome & $\begin{array}{l}\text { NLC } \\
(n=52)\end{array}$ & $\begin{array}{l}\text { PGC } \\
(n=50)\end{array}$ & $\begin{array}{l}\text { Hazard ratio } \\
\text { (one sided } 95 \% \mathrm{Cl} \text { ) }\end{array}$ & $\begin{array}{l}\text { Time ratio } \\
\text { (one sided } 95 \% \mathrm{Cl} \text { ) }\end{array}$ \\
\hline Cure at last visit or later confirmed by telephone, $n(\%)$ & $34(65.4 \%)$ & $25(50.0 \%)$ & & \\
\hline Time to event, median $(95 \% \mathrm{Cl})$, mth & $18.0(8.5$ to 27.5$)$ & 23.2 (17.3 to 29.2 ) & $1.332(0.860$ to $\infty)$ & 0.816 (0 to 1.032$)$ \\
\hline Cure at last visit, (n (\%) & $27(51.9 \%)$ & $22(44.0 \%)$ & & \\
\hline Time to event, median $(95 \% \mathrm{Cl})$, $\mathrm{mth}$ & 22.1 (15.1 to 29.2 ) & 25.1 (17.0 to 33.2 ) & $1.207(0.749$ to $\infty)$ & 0.855 (0 to 1.112$)$ \\
\hline Premature study termination, (n (\%) & $5(9.6 \%)$ & $14(28.0 \%)$ & & \\
\hline Lost to follow up & 2 & 10 & & \\
\hline Withdrew & 3 & 4 & & \\
\hline Time to event, median $(95 \% \mathrm{Cl})$, mth & $N / A^{*}$ & N/A & 0.334 (0 to 0.788$)$ & N/A $\dagger$ \\
\hline
\end{tabular}

0.85 . While our best estimate of "treatment" effect is a $33 \%$ increase in the probability of cure if the child attended the NLC rather than the PGC, a $14 \%$ lower risk of cure is not incompatible with the data. However, the balance of probability suggests that the performance of the NLC regarding cure is above acceptable limits, and is therefore as good as if not better than the PGC.

Turning our attention to the AFT model, attending the NLC hastened time to cure by a factor of 0.816 (one sided $95 \%$ CI 0 to 1.032 ) - that is, compared to the PGC, the NLC reduced time to cure by an estimated $18.4 \%$. The upper limit of the one sided $95 \%$ confidence interval equates to an increase in time to cure in the NLC of $3.2 \%$, showing equivalence well within the predefined limits of clinical equivalence of $15 \%$. Therefore, our findings suggest that children who attended the NLC were equally as likely to be cured as those attending the PGC, but their cure would occur earlier. Correspondingly, fig 1 shows a consistent advantage to the NLC over the PGC, which reinforces the non-inferiority of management of constipation undertaken by nurses when compared with doctors.

Looking at the (correlated) time to cure at last visit outcome, again more children were cured in the NLC (27/52 v $22 / 50$ in PGC) and median time to cure was reduced (22.1 $\mathrm{v}$ 25.1 months in PGC). This time, however, while having an advantage over the PGC in terms of an estimated $21 \%$ increased probability of cure. The lower confidence interval was below the threshold of clinical equivalence. However, for the AFT model, attending the NLC hastened time to cure by an estimated $14.5 \%$. The upper limit of the one sided $95 \%$ confidence interval equates to an increase in time to cure in the NLC of $11.2 \%$, showing equivalence well within the predefined limits of clinical equivalence of $15 \%$.

The precision of the results is undoubtedly affected by the reduced sample size. Had time and finances allowed we would have preferred to have recruited 100 cases into each arm of the trial. Nevertheless, analysis revealed that we had already shown what we set out too show, namely that nurses can manage intractable constipation in children at least as well as a consultant paediatric gastroenterologist.

When looking at premature study termination, a proxy measure for continuing treatment and follow up, 5 (9.6\%) children in the NLC and $14(28.0 \%)$ in the PGC were lost to follow up or withdrawn. The risk of premature study termination was significantly reduced by an estimated $66 \%$ in the NLC compared to the PGC (hazard ratio 0.33, one sided $95 \%$ CI 0 to $0.79, p=0.036$ ). Since the NLC performed significantly better than the PGC, we can confidently claim superiority of the NLC at following up the children in the study. Figure 2 clearly displays the advantage that the NLC has over the PGC regarding improving follow up; indeed from the outset the curves never cross. Once again this emphasises the non-inferiority of nurse management of this problem when compared with the medical clinic.

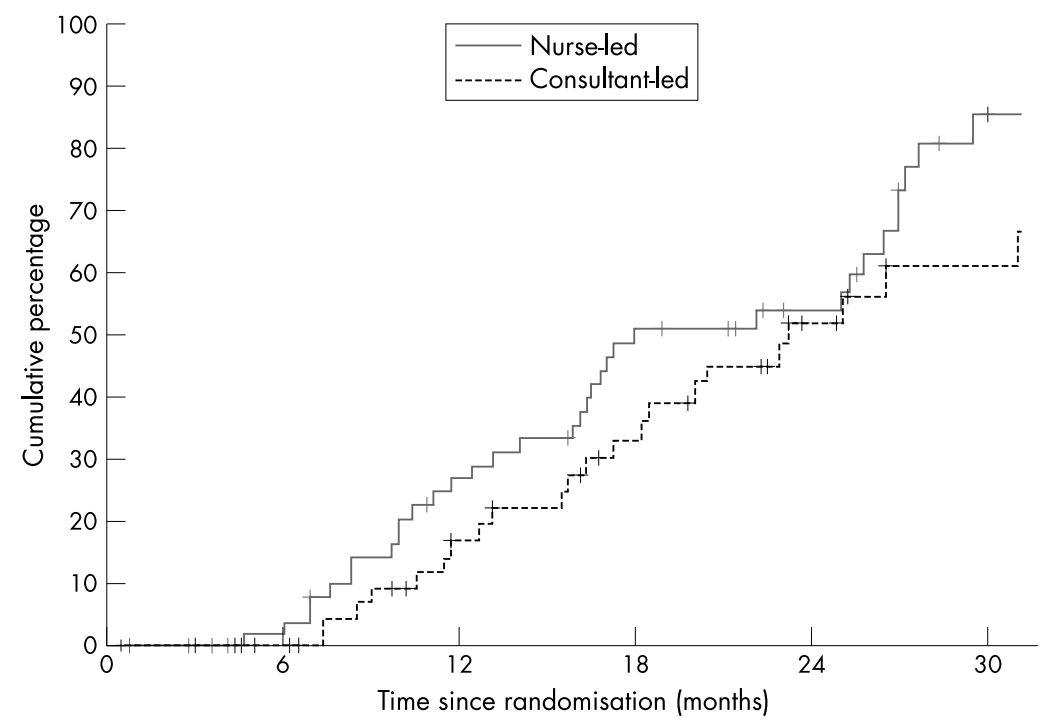

Figure 1 Percentage cured by type of clinic.

\begin{tabular}{|l|l|l|l|l|l|l|}
\hline $\begin{array}{l}\text { Number at risk } \\
\text { Nurse-led }\end{array}$ & 52 & 49 & 35 & 24 & 16 & 3 \\
\hline Consultant-led & 50 & 45 & 34 & 24 & 13 & 8 \\
\hline
\end{tabular}




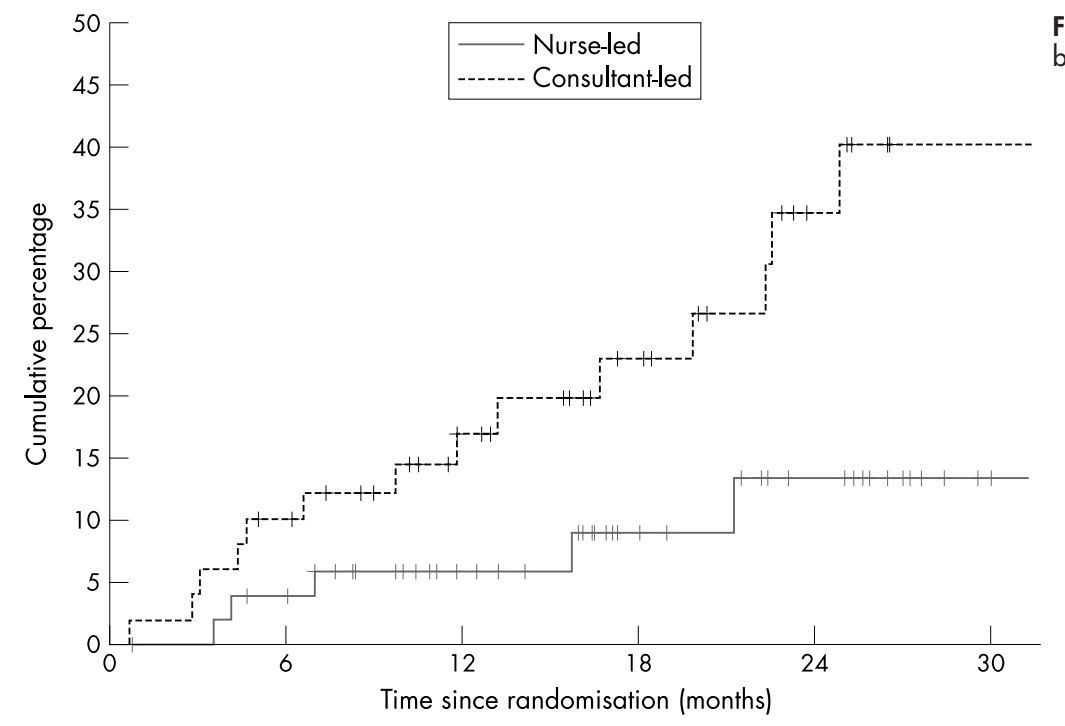

Figure 2 Percentage lost/withdrawn by type of clinic.

\begin{tabular}{|l|l|l|l|l|l|l|}
\hline $\begin{array}{l}\text { Number at risk } \\
\text { Nurse-led }\end{array}$ & 52 & 49 & 35 & 24 & 16 & 3 \\
\hline Consultant-led & 50 & 45 & 34 & 24 & 13 & 8 \\
\hline
\end{tabular}

\section{DISCUSSION}

The data in this study add to the existing literature on this subject ${ }^{2}{ }^{318}$ and provide a detailed characterisation of the clinical features found in children with intractable functional constipation. These include: the positive family history of constipation, poor dietary fibre intake, and the high prevalence of faecal soiling and voluntary faecal retention. The chronic persistence of the problem and its association with soiling often prompt referral to the tertiary centre at a time when parents are becoming worried about how the child will cope when they start school. This probably accounts for the median referral age of between $4 \frac{1}{2}$ and 5 years in our centre.

A diet low in dietary fibre was common in this group of children. Children are well known to have faddy diets and ones that are often low in fibre. The role of dietary fibre in children remains controversial, but it is a common fact that processed foods consumed by the majority of children are low in fibre, high in fat, and may well contribute to slow intestinal motility. Children with constipation often have very poor appetite and frequently "graze" over food or drink excessive amounts of milk.

Psychosocial problems associated with poor compliance in attending clinic appointments were over-represented in those children deemed not to have been cured and these data suggest that the presence of complex behavioural problems is an indicator of poor prognosis. This highlights the need for early identification of psychosocial problems and intervention by a child psychologist.

This study confirms the difficulties in the management of chronic constipation in childhood, and the overall number cured, 59/102 (58\%), may reflect the selective nature of the referral of children with intractable problems to our tertiary centre. Comparing these outcome data with other published studies is not meaningful due to the variety of study designs and the heterogeneity of the study populations. In this study, the majority of children had a history of symptoms dating back two or more years and slightly more than half of the children needed to proceed to the advanced stage of the management algorithm. This shows the association between a protracted history and the need for more intensive treatment, and highlights the importance of prevention, early detection, and early appropriate management in this group of children by primary health care professionals.

The main objective of this study was to determine whether outpatient follow up led by clinical nurse specialists with specific training in the management of childhood constipation could be delivered as effectively as, if not better than that in a consultant led paediatric gastroenterology clinic. In terms of follow up, significantly less children in the NLC were lost to follow up or withdrew from the study compared to the PGC. Nursing advice and support via the telephone was available to all children in the study as well as liaison with school and other disciplines. In terms of cure, although the difference failed to reach conventional statistical significance, a rather larger proportion of children in the NLC achieved cure than in the PGC. In addition, the survival curve suggested a consistently earlier cure in the nurse led clinic than in the medical clinic.

\section{Conclusion}

Chronic constipation in children is a common problem that may have a devastating impact on the child's life and that of the family. The management of this problem also poses a significant burden on healthcare resources. Chronic constipation in children requires multidisciplinary management and close follow up for effective outcome. The data from this study show that, when compared to outcomes from a paediatric gastroenterology clinic, trained specialist nurses provide a very effective management and follow up service.

The development of nurse led services for children with constipation is a successful concept that is becoming more popular in acute and primary care. This type of service may prove to be an ideal pathway of care for the future management of children and families with a wide range of chronic health care problems. The results of this study confirm an important role for clinical nurse specialists in the management of constipation in children and add weight to the case for expanding the role of the nurse within child healthcare.

\section{ACKNOWLEDGEMENTS}

We would like to acknowledge all the children and their parents and/ or carers, for participating in this study. We would also like to acknowledge the contributions of Jon Deeks to the design of this 
study and his support throughout, and of Michael Bradburn, who made very useful contributions to the analysis and interpretation. Special thanks go to Julia Muir who was involved in pioneering the nurse led clinic in Oxford and in the early stages of setting up this study. This study was funded by research grants from Norgine Ltd and from Wellchild.

\section{Authors' affiliations}

C A Burnett, P B Sullivan, University of Oxford, Department of Paediatrics, John Radcliffe Hospital, Oxford, UK

E Juszczak, Centre for Statistics in Medicine, Oxford, UK

\section{REFERENCES}

1 Sullivan PB. Paediatrician's approach to constipation. Curr Paediatr 1996:6:97-100.

2 Abrahamian FP, Lloyd-Still JD. Chronic constipation in childhood: a longitudinal study of 186 patients. J Pediatr Gastroenterol Nutr 1984;3:460-7.

3 Clayden GS. Management of chronic constipation. Arch Dis Child 1992:67:340-4

4 Loening-Baucke VA. Factors responsible for persistence of childhood constipation. J Pediatr Gastroenterol Nutr 1987;6:915-22.

5 Keuzenkamp-Jansen CW, Fijnvandraat CJ, Kneepkens CM, et al. Diagnostic dilemmas and results of treatment for chronic constipation. Arch Dis Child 1996:75: 1-41
6 Loening-Baucke V. Constipation in early childhood: patient characteristics, treatment, and longterm follow up. Gut 1993;34:1400-4.

7 Ferraro-McDuffie AR. The role of the pediatric clinical nurse specialist in gastroenterology. Gastroenterol Nurs 1992;14:204-7.

8 Cruikshank BM, Clow TJ, Seals B. Pediatric nurse practitioner functions in the outpatient clinics of a tertiary care center. Med Care 1986;24:4-9.

9 Muir J, Burnett CA. Setting up a nurse-led clinic for intractable childhood constipation. British Journal of Community Nursing 1999:4:395-9.

10 Jones $\mathbf{S}$. An action research investigation into the feasibility of experienced registered sick children's nurses (RSCNs) becoming children's emergency nurse practitioners (ENPs). J Clin Nurs 1996;5:1-21.

11 Royal College of Paediatrics and Child Health and the Joint Advisory Committee on Children's Nursing. Developing Roles of Nurses in Clinical Child Health. Report from a joint working party, 1996.

12 Felt B, Wise CG, Olson A, et al. Guideline for the management of pediatric idiopathic constipation and soiling. Multidisciplinary team from the University of Michigan Medical Center in Ann Arbor. Arch Pediatr Adolesc Med 1999; 153:380-5

13 Baker SS, Liptak GS, Colletti RB, et al. Constipation in infants and children: evaluation and treatment. A medical position statement of the North American Society for Pediatric Gastroenterology and Nutrition. J Pediatr Gastroenterol Nutr 1999;29:5-26.

14 Poenaru D, Roblin N, Bird M, et al. The Pediatric Bowel Management Clinic: initial results of a multidisciplinary approach to functional constipation in children. J Pediatr Surg 1997:32:6-8.

15 Leech SC, McHugh K, Sullivan PB. Evaluation of a method of assessing faecal loading on plain abdominal radiographs in children. Pediatr Radiol $1999 ; 29: 255-8$.

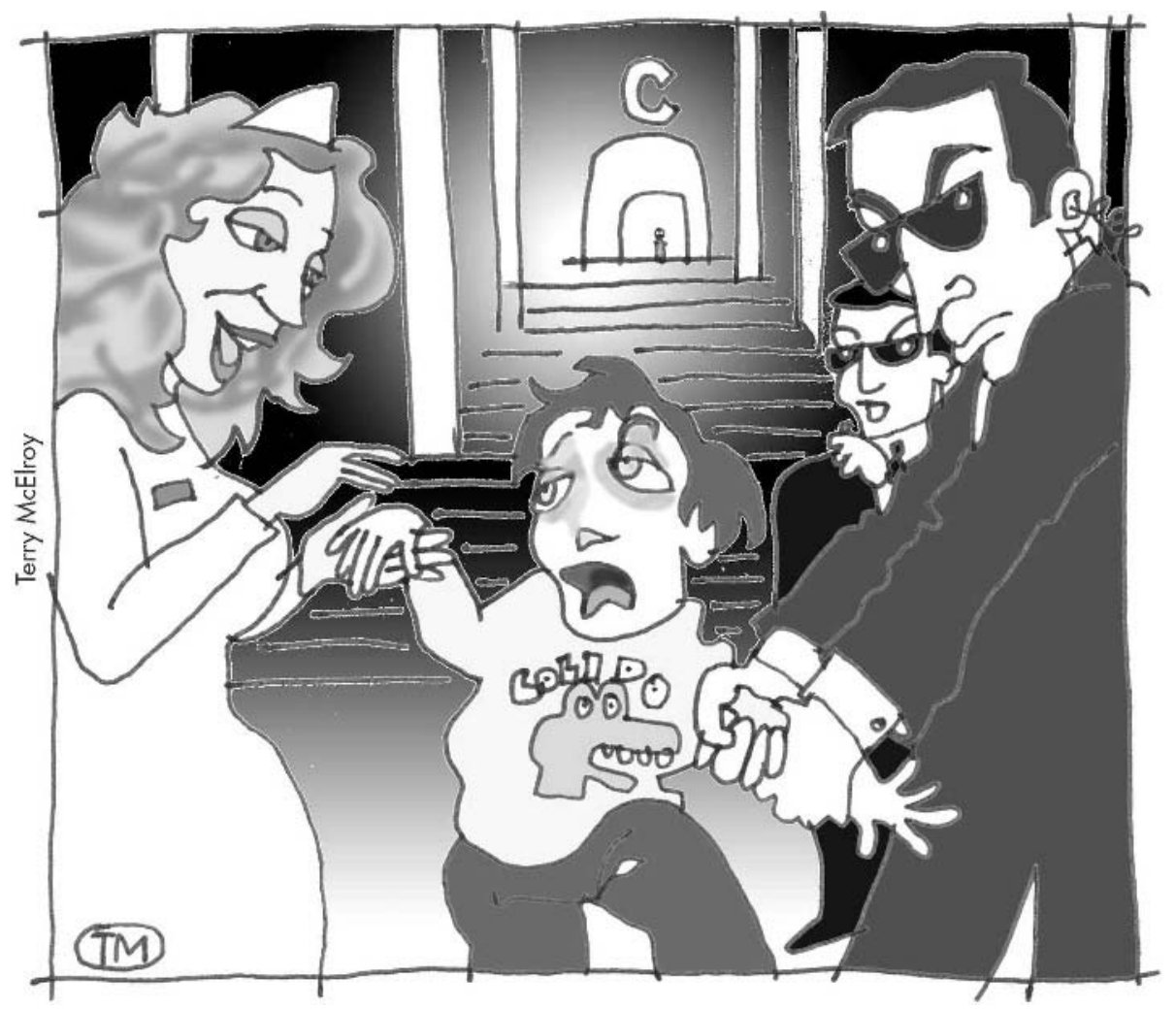

